\title{
ELASTICITY IN LIQUIDS
}

A SYMPOSIUM on elasticity in liquids, arranged under the auspices of the British Society of Rheology, was held on March 27 in the Department of Science of the Durham Colleges in the University of Durham. Mr. J. E. Caffyn organized the details of the meeting.

Prof. J. E. P. Wagstaff welcomed the members to Durham, and the morning session then opened with Prof. G. S. Rushbrooke in the chair. Dr. R. Roscoe first read a paper on elastic relaxation and flow in suspensions, in which he referred to the early experiments of Schwedoff on relaxing sols. The method involved placing a gelatine solution in a co-axial cylinder viscometer, suddenly applying a shear by twisting the torsion head which supported the inner cylinder and observing the stress on the system as it relaxed slowly. Schwedoff considered that the stress fell off exponentially; but later workers do not confirm this. Dr. Roscoe has found, however, by repeating their experiments, that departure from the exponential decay is a feature of viscometers with a wide gap between the cylinders. By writing down the flow equations for such an apparatus, he is able to deduce a form of decay for the stress which agrees with experiment.

In the second part of his paper, Dr. Roscoe discussed the relation between rate of shear and shear stress, under steady conditions. Equations of flow have been developed by a generalization of Maxwell's method, which reduce to Bingham's equation in the case of steady flow. The cause of observed departures from Bingham's equation was discussed.

Dr. S. Thornton, speaking on the rheological behaviour of some dispersions of solid particles in liquid media, considered the effect of particle concentration on the relative viscosity of suspensions of rigid non-flocculating particles, and he developed a general expression applicable not only to spherical particles, in agreement with Einstein's well-known equation, but also to non-spherical particles of a limited type over a limited range of concentration. After a brief review of the experimental work on this subject, he stressed the need for checking the absence of flocculation of the particles and for ensuring the validity of any corrections applied. He explained briefly the technique for measuring the absolute viscosity of thixotropic materials which do not exhibit elastic behaviour, and gave results for some special cases.

Dr. E. G. Richardson described some experiments now in progress on the relationship between shear and bulk viscosity. Stokes first deduced a formula for the absorption of plane waves in a gas, the theory making the coefficient $(\alpha)$ proportional to the square of the frequency $(f)$ and the kinematic viscosity $(v)$. In many liquids $\alpha / f^{2}$ is constant (with frequency) but has a value much greater than that of Stokes's formula, if the shear viscosity is substituted for $v$. It has been suggested that the bulk, or dilatational viscosity, should bo included in $\nu$ when the propagation of ultrasonic waves is concerned. One experiment, suggested by Eckart, for the determination of bulk viscosity is based on the mensuration of the acoustic streams in the neighbourhood of a vibrating source, and a study of this is being made using hot wires to measure the streaming velocities in various fluids at frequencies from $1 \mathrm{c} . / \mathrm{s}$. to $1 \mathrm{Mc} . / \mathrm{s}$.
Another method is to study the absorption of ultrasonics in capillary tubes, wherein plane waves are propagated in the centre of the tube and shear waves in the acoustic boundary-layer near the walls. Some initial results in both experiments were quoted.

In the afternoon session, with Dr. J. G. Oldroyd in the chair, J. E. Caffyn and R. M. Underwood described a new method for delineating velocity profiles in visco-elastic liquids. This involves the delineation of the motion of isolated oil drops, suspended in the liquid under test flowing along a tube $1 \mathrm{~cm}$. in diameter, by double-flash photography from two perpendicular directions. From the double exposure of the oil drop on the photograph, the velocity at different radial distances from the axis of the tube can be estimated (and incidentally any deviation from axial flow detected), and hence the velocity profile at the site of the photographs to be constructed. Examples in water (for calibration purposes) and in an ammonium oleate sol were shown. In the visco-elastic fluid the velocity distribution, in accordance with the theories of oldroyd and of Reiner and Rivlin, is still parabolic.

The final paper, by Dr. A. S. Lodge, considered some theories of the Weissenberg effect, and dealt with the distribution of transverse pressure in a rotating cone-and-plate viscometer, when the interspace is filled with a visco-elastic liquid in which components of stress perpendicular to the direction of shear are set up. The paper was theoretical and dealt with the fluid in stationary laminar incompressible flow, for which the general stress determinant was constructed. The difference between stress components (zero in a Newtonian fluid) give rise to the Weissenberg effect, as Dr. Lodge showed. Having calculated these differences for various radial distances in the coneand-plate apparatus, he can compare the theory with the measurements of Roberts, who erected a series of manometers from the centre to the rim of the cone above the rotating plate and so was able to measure the transverse (vertical) stress distribution in a number of visco-elastic liquids. In the discussion on this paper, Dr. K. Weissenberg pointed out that Roborts's experimental results agree with his own calculation of the stress distribution.

\section{MATHEMATICAL ASSOCIATION}

\section{ANNUAL GENERAL MEETING IN SHEFFIELD}

T HE annual general meeting of the Mathematical Association was held in the University of Sheffield during April 8-11. At a reception given by the University on April 8, Dr. J. M. Whittaker, the vice-chancellor, welcomed the members, among whom was his father, Sir Edmund Whittaker, honorary member of the Association and president in 1919.

On April 9, following the business meeting, the president, Mr. K. S. Snell (Harrow School), gave his address, his subject being "School Mathematics To-day and To-morrow". Mr. Snell began with a speculation on the probable content of the school curriculum fifty years hence, suggesting that many topics now regarded as well outside the school range would by then have become commonplaces of the 